International Mathematical Forum, 1, 2006, no. 31, 1497 - 1509

\title{
FREQUENTLY OSCILLATORY SOLUTIONS FOR MULTI-LEVEL PARTIAL DIFFERENCE EQUATIONS
}

\author{
Zhi-Qiang Zhu \\ Department of Computer Science \\ Guangdong Polytechnic Normal University \\ Guangzhou 510665, P. R. China \\ Sui Sun Cheng \\ Department of Mathematics \\ Tsing Hua University \\ Hsinchu, Taiwan 30043, R. O. China
}

\begin{abstract}
Multi-level partial difference equations arise in many mathematical models. By introducing frequency measures for double sequences defined on half lattice planes, oscillatory solutions can be defined for these equations and frequent oscillation criteria found.
\end{abstract}

Mathematics Subject Classification: 39A10

Keywords: asymptotic density, frequency measure, frequent oscillation, multilevel partial difference equation

\section{Introduction}

In [1], the two-level partial difference equation

$$
u_{m}^{(n+1)}=a u_{m-1}^{(n)}+b u_{m}^{(n)}+c u_{m+1}^{(n)}, m \in Z, n \in N
$$

where $a, b, c \in R, Z$ is the set of integers and $N$ the set of nonnegative integers, is considered. Such an equation arises in several mathematical models (see e.g. [2]) including heat transfer in lattice of molecules, population migration among cities, discrete simulation of the heat equation, etc. 
Note that solutions of (1) of the form $\left\{u_{m}^{(n)}\right\}_{m \in Z, n \in N}$ exist and depend uniquely on the initial distribution $\left\{u_{m}^{(0)}\right\}_{m \in Z}$ and can be computed successively by

$$
u_{0}^{(1)} ; u_{-1}^{(1)}, u_{1}^{(1)}, u_{0}^{(2)} ; u_{-2}^{(1)}, u_{-1}^{(2)}, u_{2}^{(1)}, u_{1}^{(2)}, u_{0}^{(3)} ; \ldots
$$

As an example, suppose $b=0$ in (1). Let $u_{m}^{(0)}=f_{m}$ for $m \in Z$, where $f_{m}=0$ when $m$ is odd and $f_{m}=(-1)^{m / 2}$ when $m$ is even. Then by means of the computational scheme stated above, we obtain the sequence

$$
0 ;-(a-c),(a-c),-(a-c)^{2} ; 0,0,0,0,0 ;(a-c),(a-c)^{2},(a-c)^{3}, \ldots,
$$

which shows that $u_{m}^{(n)}$ is either equal to 0 or to $\pm(a-c)^{n}$. In particular, if $a>c$, then the corresponding solution is seen to be 'oscillatory' in the sense that there are always positive terms and negative terms of our solution in any 'exterior domain' defined by $|m| \geq m_{0}$ and $n \geq n_{0}$. This prompts us to consider the existence of oscillatory solutions for more general partial difference equations.

Let us denote the set of all integers by $Z$, the set of nonnegative integers by $N$, the set $\{a, a+1, \ldots, b\}$ of integers by $Z[a, b]$, and the set $\{a, a+1, a+2, \ldots\}$ of integers by $Z[a, \infty)$. In this paper, we will study a multi-level partial difference equation of the form

$$
u_{m}^{(n+1)}+a_{m}^{(n)} u_{m+k}^{(n)}-b_{m}^{(n)} u_{m}^{(n-\delta)}-c_{m}^{(n)} u_{m-l}^{(n)}=0,(m, n) \in Z \times N,
$$

where $\left\{a_{m}^{(n)}\right\},\left\{b_{m}^{(n)}\right\}$ and $\left\{c_{m}^{(n)}\right\}$ are real double sequences defined on $Z \times N$, $k, l$ are positive integers and $\delta$ is a nonnegative integer. Again, when initial distributions $\left\{u_{m}^{(-\delta)}\right\}_{m \in Z}, \ldots,\left\{u_{m}^{(-1)}\right\}_{m \in Z}$ and $\left\{u_{m}^{(0)}\right\}_{m \in Z}$ are given, it is possible to calculate a unique solution $\left\{u_{m}^{(n)}\right\}$ of $(2)$, defined for $(m, n) \in Z \times Z[-\delta, \infty)$, by slightly modifying the scheme described above. But the existence and nonexistence of oscillatory solutions will need careful mathematical investigation. In this paper, we will make use of the concept of asymptotic density (also known as frequency measure) for subsets of the lattice set $Z \times Z[-\delta, \infty)$ to define oscillatory double sequences of the form $\left\{x_{m}^{(n)}\right\}_{(m, n) \in Z \times Z[-\delta, \infty)}$. Oscillation criteria are then derived for solutions of (2).

A sequence $A$ of positive integers $a_{1}<a_{2}<\cdots$ has lower asymptotic density $\underline{\delta}(A)$ and upper asymptotic density $\bar{\delta}(A)$ defined by

$$
\underline{\delta}(A)=\liminf _{n \rightarrow \infty} \frac{A(n)}{n}, \bar{\delta}(A)=\limsup _{n \rightarrow \infty} \frac{A(n)}{n},
$$

where $A(n)$ denotes the number of integers of $A$ which are not greater than $n$. These concepts and related ones such as the Schnirelmann density of $A$ have 
been extensively used in number theory for stating properties of integer sequences (see e.g. [3,4]). In [5] and [6], the authors discover that these densities for subsets of $\left\{k \in Z \mid k \geq k_{0}\right\}$ and subsets of $\left\{(m, n) \in Z^{2} \mid m \geq m_{0}, n \geq n_{0}\right\}$ can also be used to discuss the oscillatory properties of real sequences and double sequences. Since the emphasis is changed to the values of sequences (instead of their domains of definition), asymptotic densities are called frequency measures.

To the best of our knowledge, this is the first time oscillation of the multilevel partial difference equation (2) is discussed by employing frequency measures.

\section{Frequency Measures}

There are many results related to frequency measures, the ones we need in this paper, however, are limited. For the sake of completeness, the definitions of frequency measures and their elementary properties will be briefly sketched as follows.

The union, intersection of two sets $A$ and $B$ will be denoted by $A+B$ and $A \cdot B$ respectively, while $A-B$ denotes the relative complement of $B$ in $A$. Let $\Omega=Z \times Z[-\delta, \infty)$ and let $l^{\Omega}$ be the set of all real double sequences of the form $\left\{u_{m}^{(n)}\right\}_{(m, n) \in \Omega}$. Let $\Phi$ be a subset of $\Omega$. The number of elements of $\Phi$ will be denoted by $|\Phi|$. Given an integer $m$, the translation operators $X^{m}$ and $Y^{m}$ will be defined respectively by

$$
X^{m} \Phi=\{(i+m, j) \in \Omega:(i, j) \in \Phi\}
$$

and

$$
Y^{m} \Phi=\{(i, j+m) \in \Omega:(i, j) \in \Phi\} .
$$

Let $\alpha, \beta, \lambda$ and $\tau$ be integers satisfying $\alpha \leq \beta$ and $\lambda \leq \tau$. The union $\sum_{i=\alpha}^{\beta} \sum_{j=\lambda}^{\tau} X^{i} Y^{j} \Phi$ will be denoted by $X_{\alpha}^{\beta} Y_{\lambda}^{\tau} \Phi$. Clearly, for any lattice point $(i, j) \in \Omega$ with $j \geq \tau-\delta$, we have

$$
(i, j) \in \Omega-X_{\alpha}^{\beta} Y_{\lambda}^{\tau} \Phi \Leftrightarrow(i-s, j-t) \in \Omega-\Phi
$$

for $\alpha \leq s \leq \beta$ and $\lambda \leq t \leq \tau$. For any nonnegative integers $m$ and $n$, we set

$$
\Phi^{(m, n)}=\{(i, j) \in \Phi:|i| \leq m \text { and }-\delta \leq j \leq n\} .
$$

Definition 2.1 Let $\Phi$ be a subset of $\Omega$. If

$$
\limsup _{m, n \rightarrow \infty} \frac{\left|\Phi^{(m, n)}\right|}{2 m n}
$$


exists, then this limit, denoted by $\mu^{*}(\Phi)$, will be called the upper frequency measure of $\Phi$. Similarly, if

$$
\liminf _{m, m \rightarrow \infty} \frac{\left|\Phi^{(m, n)}\right|}{2 m n}
$$

exists, this limit, denoted by $\mu_{*}(\Phi)$, will be called the lower frequency measure of $\Phi$. If $\mu^{*}(\Phi)=\mu_{*}(\Phi)$, then the common limit denoted by $\mu(\Phi)$ will be called the frequency measure of $\Phi$.

As immediate examples, $\mu(\emptyset)=0$ and $\mu(\Omega)=1$. As another example, let $X=\left\{n \in Z\left[2^{i}, 2^{i}+2^{i-1}\right]: i \in Z[1, \infty)\right\}$ and $\Phi=\{(i, j): \pm i \in X, j \in X\}$. Then $\Phi \subset \Omega,\left|\Phi^{\left(2^{n}, 2^{n}\right)}\right|=2\left(2^{n-1}+n-1\right)^{2}$ and $\left|\Phi^{\left(2^{n}+2^{n-1}, 2^{n}+2^{n-1}\right)}\right|=2\left(2^{n}+n-1\right)^{2}$ for each positive integer $n$. Hence, we have

$$
\frac{\left|\Phi^{\left(2^{n}, 2^{n}\right)}\right|}{2 \times 2^{2 n}}=\left(\frac{2^{n-1}+n-1}{2^{n}}\right)^{2} \rightarrow \frac{1}{4} \text { as } n \rightarrow \infty
$$

and

$$
\frac{\left|\Phi^{\left(2^{n}+2^{n-1}, 2^{n}+2^{n-1}\right)}\right|}{2\left(2^{n}+2^{n-1}\right)^{2}}=\left(\frac{2^{n}+n-1}{2^{n}+2^{n-1}}\right)^{2} \rightarrow \frac{4}{9} \text { as } n \rightarrow \infty .
$$

Thus $\mu_{*}(\Phi) \leq 1 / 4$ and $\mu^{*}(\Phi) \geq 4 / 9$.

The following results are concerned with the properties of frequency measures and their proofs are similar to those in [2].

Lemma 2.2 Let $\Phi$ and $\Gamma$ be subsets of $\Omega$. Then

(i) if $\Phi \subseteq \Gamma$, then $\mu^{*}(\Phi) \leq \mu^{*}(\Gamma)$ and $\mu_{*}(\Phi) \leq \mu_{*}(\Gamma)$;

(ii) $\mu_{*}(\Phi)+\mu^{*}(\Gamma)-\mu^{*}(\Phi \cdot \Gamma) \leq \mu^{*}(\Phi+\Gamma) \leq \mu^{*}(\Phi)+\mu^{*}(\Gamma)-\mu_{*}(\Phi \cdot \Gamma)$;

(iii) $\mu_{*}(\Phi)+\mu_{*}(\Gamma)-\mu^{*}(\Phi \cdot \Gamma) \leq \mu_{*}(\Phi+\Gamma) \leq \mu_{*}(\Phi)+\mu^{*}(\Gamma)-\mu_{*}(\Phi \cdot \Gamma)$;

(iv) $\mu_{*}(\Phi)+\mu^{*}(\Omega-\Phi)=1$;

(v) if $\Phi \cdot \Gamma$ is finite, then $\mu^{*}(\Phi)+\mu_{*}(\Gamma) \leq 1$.

Lemma 2.3 Let $\Phi$ be a subset of $\Omega$ and $\alpha, \beta, \lambda$ and $\tau$ be integers such that $\alpha \leq \beta$ and $\lambda \leq \tau$. Then

$$
\mu^{*}\left(X_{\alpha}^{\beta} Y_{\lambda}^{\tau} \Phi\right) \leq(\beta-\alpha+1)(\tau-\lambda+1) \mu^{*}(\Phi)
$$

and

$$
\mu_{*}\left(X_{\alpha}^{\beta} Y_{\lambda}^{\tau} \Phi\right) \leq(\beta-\alpha+1)(\tau-\lambda+1) \mu_{*}(\Phi) .
$$


As an immediate consequence of Lemma 2.2 (i) to (iii) and mathmatical induction, if $\Phi_{1}, \ldots, \Phi_{n}$ are subsets of $\Omega$, then

$$
\mu^{*}\left(\sum_{i=1}^{n} \Phi_{i}\right) \leq \sum_{i=1}^{n} \mu^{*}\left(\Phi_{i}\right)-(n-1) \mu_{*}\left(\prod_{i=1}^{n} \Phi_{i}\right)
$$

and

$$
\mu_{*}\left(\sum_{i=1}^{n} \Phi_{i}\right) \leq \mu_{*}\left(\Phi_{1}\right)+\sum_{i=2}^{n} \mu^{*}\left(\Phi_{i}\right)-(n-1) \mu_{*}\left(\prod_{i=1}^{n} \Phi_{i}\right)
$$

Let $u=\left\{u_{m}^{(n)}\right\}_{(m, n) \in \Omega}, v=\left\{v_{m}^{(n)}\right\}_{(m, n) \in \Omega} \in l^{\Omega}$ and $c_{1}, c_{2} \in R$. The set $\left\{(m, n) \in \Omega: u_{m}^{(n)} \geq c_{1}\right\}$ will be denoted by $\left(u \geq c_{1}\right)$. The notations $\left(u>c_{1}\right)$, $\left(u<c_{1}\right),\left(u \leq c_{1}\right)$ are similarly defined. By these notations, $\left(u \leq c_{1}\right)+\left(v \leq c_{2}\right)$ then stands for $\left\{(m, n) \in \Omega: u_{m}^{(n)} \leq c_{1}\right.$ or $\left.v_{m}^{(n)} \leq c_{2}\right\}$, etc.

Double sequences in $l^{\Omega}$ may exhibit many different oscillatory behaviors. For instance, we may say that a double sequence $\left\{u_{m}^{(n)}\right\}_{(m, n) \in \Omega}$ is eventually positive (or negative) if $u_{m}^{(n)}>0$ (or $u_{m}^{(n)}<0$ ) for all large $|m|$ and $n$. We may say that it is oscillatory if it is neither eventually positive nor eventually negative. In this paper, we introduce a few more concepts related to the oscillatory behaviors of double sequences in $l^{\Omega}$.

Definition 2.4 Let $u=\left\{u_{m}^{(n)}\right\}_{(m, n) \in \Omega} \in l^{\Omega}$. If $\mu^{*}(u \leq 0)=0$, then $u$ is said to be frequently positive. If $\mu^{*}(u \geq 0)=0$, then $u$ is said to be frequently negative. The double sequence $u$ is said to be frequently oscillatory if $u$ is neither frequently positive nor frequently negative.

Definition 2.5 Let $u=\left\{u_{m}^{(n)}\right\}_{(m, n) \in \Omega} \in l^{\Omega}$. If $\mu^{*}(u>0)=\omega \in(0,1)$, then $u$ is said to have unsaturated upper positive part. If $\mu_{*}(u>0)=\omega \in(0,1)$, then $u$ is said to have unsaturated lower positive part. The double sequence $u$ is said to have unsaturated positive part if $\mu^{*}(u>0)=\mu_{*}(u>0)=\omega \in(0,1)$.

We remark that a sequence which has unsaturated negative part can similarly be defined. For the sake of simplicity, we will restrict our attentions to the sequences with unsaturated positive part.

Note that if a double sequence $u$ is frequently oscillatory, then it is oscillatory. Furthermore, in case $u$ is eventually positive, then $\mu(u>0)=1$. And, in case $u$ is eventually negative, then $\mu(u>0)=0$. Hence, if a double sequence $u$ has unsaturated upper (or lower) positive part, then it is oscillatory. 


\section{Oscillation Criteria}

As before, we set $\Omega=Z \times Z[-\delta, \infty)$. Throughout this section, for any double sequence $\left\{u_{m}^{(n)}\right\}_{(m, n) \in \Omega}$, we also set

$$
\begin{gathered}
\tilde{u}_{m}^{(n)}=\sum_{i=m-l}^{m+k-1} \sum_{j=n-\delta}^{n} u_{i}^{(j)}, \\
\underline{u}_{m}^{(n)}=\min \left\{u_{i}^{(j)}: m-l \leq i \leq m+k-1 \text { and } n-\delta \leq j \leq n\right\}
\end{gathered}
$$

as well as

$$
\bar{u}_{m}^{(n)}=\max \left\{u_{i}^{(j)}: m-l \leq i \leq m+k-1 \text { and } n-\delta \leq j \leq n\right\}
$$

for $(m, n) \in Z \times N$.

Lemma 3.1 Suppose there exists $\left(m_{0}, n_{0}\right) \in \Omega$ with $n_{0}-3 \delta \geq 0$ such that $a_{m}^{(n)} \geq 1, b_{m}^{(n)} \leq 0, c_{m}^{(n)} \leq 0,(m, n) \in Z\left[m_{0}-3 l, m_{0}+2 k-1\right] \times Z\left[n_{0}-3 \delta, n_{0}+1\right]$.

Let $\left\{u_{m}^{(n)}\right\}_{(m, n) \in \Omega}$ be a solution of (2). If $u_{m}^{(n)} \geq 0$ on $Z\left[m_{0}-3 l, m_{0}+2 k-1\right] \times$ $Z\left[n_{0}-3 \delta, n_{0}+1\right]$, then

$$
\Delta^{(1,0)} \tilde{u}_{m}^{(n)} \leq 0, \Delta^{(0,1)} \tilde{u}_{m}^{(n)} \leq 0,(m, n) \in Z\left[m_{0}-l, m_{0}\right] \times Z\left[n_{0}-\delta, n_{0}\right],
$$

and if $u_{m}^{(n)} \leq 0$ on $Z\left[m_{0}-3 l, m_{0}+2 k-1\right] \times Z\left[n_{0}-3 \delta, n_{0}+1\right]$, then

$$
\Delta^{(1,0)} \tilde{u}_{m}^{(n)} \geq 0, \Delta^{(0,1)} \tilde{u}_{m}^{(n)} \geq 0,(m, n) \in Z\left[m_{0}-l, m_{0}\right] \times Z\left[n_{0}-\delta, n_{0}\right],
$$

where $\Delta^{(1,0)} \tilde{u}_{m}^{(n)}=\tilde{u}_{m+1}^{(n)}-\tilde{u}_{m}^{(n)}$ and $\Delta^{(0,1)} \tilde{u}_{m}^{(n)}=\tilde{u}_{m}^{(n+1)}-\tilde{u}_{m}^{(n)}$.

Proof. In view of (5), we see that

$$
\Delta^{(1,0)} \tilde{u}_{m}^{(n)}=\sum_{i=m+1-l}^{m+k} \sum_{j=n-\delta}^{n} u_{i}^{(j)}-\sum_{i=m-l}^{m+k-1} \sum_{j=n-\delta}^{n} u_{i}^{(j)}=\sum_{j=n-\delta}^{n} u_{m+k}^{(j)}-\sum_{j=n-\delta}^{n} u_{m-l}^{(j)} .
$$

In view of (2), we also have

$$
u_{m+k}^{(j)}-u_{m-l}^{(j)}=\left(1-a_{m}^{(j)}\right) u_{m+k}^{(j)}+b_{m}^{(j)} u_{m}^{(j-\delta)}+\left(c_{m}^{(j)}-1\right) u_{m-l}^{(j)}-u_{m}^{(j+1)} .
$$


Thus

$$
\Delta^{(1,0)} \tilde{u}_{m}^{(n)}=\sum_{j=n-\delta}^{n}\left(\left(1-a_{m}^{(j)}\right) u_{m+k}^{(j)}+b_{m}^{(j)} u_{m}^{(j-\delta)}+\left(c_{m}^{(j)}-1\right) u_{m-l}^{(j)}-u_{m}^{(j+1)}\right) .
$$

Similarly, we have

$$
\Delta^{(0,1)} \tilde{u}_{m}^{(n)}=\sum_{i=m-l}^{m+k-1}\left(-a_{i}^{(n)} u_{i+k}^{(n)}+\left(b_{i}^{(n)}-1\right) u_{i}^{(n-\delta)}+c_{i}^{(n)} u_{i-l}^{(n)}\right) .
$$

Invoking the assumptions in (8), we may easily check that our assertions hold. The proof is complete.

Lemma 3.2 Let $\left(m_{0}, n_{0}\right) \in \Omega$ with $n_{0}-3 \delta \geq 0$, and let $\left\{u_{m}^{(n)}\right\}_{(m, n) \in \Omega}$ be a solution of (2). If $u_{m}^{(n)} \geq 0$ on $Z\left[m_{0}-3 l, m_{0}+2 k-1\right] \times Z\left[n_{0}-3 \delta, n_{0}+1\right]$, then

$$
\begin{gathered}
\tilde{u}_{m}^{(n+1)}+\underline{a}_{m}^{(n)} \tilde{u}_{m+k}^{(n)}-\bar{b}_{m}^{(n)} \tilde{u}_{m}^{(n-\delta)}-\bar{c}_{m}^{(n)} \tilde{u}_{m-l}^{(n)} \leq 0, \\
(m, n) \in Z\left[m_{0}-l, m_{0}\right] \times Z\left[n_{0}-\delta, n_{0}\right],
\end{gathered}
$$

and if $u_{m}^{(n)} \leq 0$ on $Z\left[m_{0}-3 l, m_{0}+2 k-1\right] \times Z\left[n_{0}-3 \delta, n_{0}+1\right]$, then

$$
\begin{gathered}
\tilde{u}_{m}^{(n+1)}+\underline{a}_{m}^{(n)} \tilde{u}_{m+k}^{(n)}-\bar{b}_{m}^{(n)} \tilde{u}_{m}^{(n-\delta)}-\bar{c}_{m}^{(n)} \tilde{u}_{m-l}^{(n)} \geq 0, \\
(m, n) \in Z\left[m_{0}-l, m_{0}\right] \times Z\left[n_{0}-\delta, n_{0}\right] .
\end{gathered}
$$

Proof. By (2) and (5),we have for $u_{m}^{(n)} \geq 0$ on $Z\left[m_{0}-3 l, m_{0}+2 k-1\right] \times$ $Z\left[n_{0}-3 \delta, n_{0}+1\right]$ that

$$
\begin{aligned}
0 & =\sum_{i=m-l}^{m+k-1} \sum_{j=n-\delta}^{n}\left(u_{i}^{(j+1)}+a_{i}^{(j)} u_{i+k}^{(j)}-b_{i}^{(j)} u_{i}^{(j-\delta)}-c_{i}^{(j)} u_{i-l}^{(j)}\right) \\
& \geq \tilde{u}_{m}^{(n+1)}+\underline{a}_{m}^{(n)} \tilde{u}_{m+k}^{(n)}-\bar{b}_{m}^{(n)} \tilde{u}_{m}^{(n-\delta)}-\bar{c}_{m}^{(n)} \tilde{u}_{m-l}^{(n)},
\end{aligned}
$$

where we have used the obvious fact that $\underline{a}_{m}^{n} \leq a_{i}^{j}, \bar{b}_{m}^{n} \geq b_{i}^{j}$ and $\bar{c}_{m}^{n} \geq c_{i}^{j}$ for $m-l \leq i \leq m+k-1$ and $n-\delta \leq j \leq n$. (10) is similarly proved. The proof is complete.

Theorem 3.3 Suppose there exist constants $\omega_{a}, \omega_{b}, \omega_{c}$ and $\omega$ such that $\mu^{*}(a<$ 1) $=\omega_{a}, \mu^{*}(b>0)=\omega_{b}, \mu^{*}(c>0)=\omega_{c}$, $\mu_{*}((a<1) \cdot(b>0) \cdot(c>0))=\omega$ and

$$
\mu_{*}(\bar{b}<-\bar{c})>(2 k+3 l)(3 \delta+2)\left(\omega_{a}+\omega_{b}+\omega_{c}-2 \omega\right) .
$$

Then every nontrivial solution $u=\left\{u_{m}^{(n)}\right\}$ of (2) is frequently oscillatory. 
Proof. Suppose to the contrary that $u$ is eventually positive. Then $\mu^{*}(u \leq$ $0)=0$. By Lemma 2.2(iv), Lemma 2.3 and (4), we have

$$
\begin{aligned}
1= & \mu^{*}\left(\Omega-X_{-2 k+1}^{3 l} Y_{-1}^{3 \delta}((a<1)+(b>0)+(c>0)+(u \leq 0))\right) \\
& +\mu_{*}\left(X_{-2 k+1}^{3 l} Y_{-1}^{3 \delta}((a<1)+(b>0)+(c>0)+(u \leq 0))\right) \\
\leq & \mu^{*}\left(\Omega-X_{-2 k+1}^{3 l} Y_{-1}^{3 \delta}((a<1)+(b>0)+(c>0)+(u \leq 0))\right) \\
& +(2 k+3 l)(3 \delta+2)\left\{\mu_{*}((a<1)+(b>0)+(c>0))+\mu^{*}(u \leq 0)\right\} \\
\leq & \mu^{*}\left(\Omega-X_{-2 k+1}^{3 l} Y_{-1}^{3 \delta}((a<1)+(b>0)+(c>0)+(u \leq 0))\right) \\
& +(2 k+3 l)(3 \delta+2)\left(\omega_{a}+\omega_{b}+\omega_{c}-2 \omega\right) \\
< & \mu^{*}\left(\Omega-X_{-2 k+1}^{3 l} Y_{-1}^{3 \delta}((a<1)+(b>0)+(c>0)+(u \leq 0))\right)+\mu_{*}(\bar{b}<-\bar{c}) .
\end{aligned}
$$

Hence, by Lemma 2.2(v),

$$
\left(\Omega-X_{-2 k+1}^{3 l} Y_{-1}^{3 \delta}((a<1)+(b>0)+(c>0)+(u \leq 0))\right) \cdot(\bar{b}<-\bar{c})
$$

is an infinite subset of $\Omega$. This, together with (3), mean that there exists $\left(m_{0}, n_{0}\right) \in \Omega$ with $n_{0}-3 \delta \geq 0$ such that

$$
\bar{b}_{m_{0}}^{n_{0}}<-\bar{c}_{m_{0}}^{n_{0}}
$$

and for $(m, n) \in Z\left[m_{0}-3 l, m_{0}+2 k-1\right] \times Z\left[n_{0}-3 \delta, n_{0}+1\right]$,

$$
a_{m}^{(n)} \geq 1, b_{m}^{(n)} \leq 0, c_{m}^{(n)} \leq 0, u_{m}^{(n)}>0 .
$$

By Lemma 3.2, associated with (12), we see that (9) holds for $m_{0}-l \leq m \leq m_{0}$ and $n_{0}-\delta \leq n \leq n_{0}$. Invoking (12) again, from Lemma 3.1 and (9), we have for $m=m_{0}$ and $n=n_{0}$ that

$$
0 \geq \tilde{u}_{m}^{(n+1)}+\underline{a}_{m}^{(n)} \tilde{u}_{m+k}^{(n)}-\bar{b}_{m}^{(n)} \tilde{u}_{m}^{n-\delta}-\bar{c}_{m}^{(n)} \tilde{u}_{m-l}^{(n)} \geq\left(-\bar{b}_{m}^{(n)}-\bar{c}_{m}^{(n)}\right) \tilde{u}_{m}^{(n)},
$$

where we have imposed $\tilde{u}_{m}^{(n+1)}>0, \tilde{u}_{m+k}^{(n)}>0, \underline{a}_{m}^{(n)} \geq 1, \bar{b}_{m}^{(n)} \leq 0$ and $\bar{c}_{m}^{(n)} \leq 0$ for $m=m_{0}$ and $n=n_{0}$. From (13), we find $\bar{b}_{m_{0}}^{\left(n_{0}\right)} \geq-\bar{c}_{m_{0}}^{\left(n_{0}\right)}$, which is contrary to $(11)$.

In case $u$ is eventually negative, then $\mu^{*}(u \geq 0)=0$. By arguments similar to those above, we may see that

$$
\left(\Omega-X_{-2 k+1}^{3 l} Y_{-1}^{3 \delta}((a<1)+(b>0)+(c>0)+(u \geq 0))\right) \cdot(\bar{b}<-\bar{c})
$$

is an infinite subset of $\Omega$. There then exists $\left(m_{0}, n_{0}\right) \in \Omega$ with $n_{0}-3 \delta \geq 0$ such that (11) and

$$
a_{m}^{n} \geq 1, b_{m}^{n} \leq 0, c_{m}^{n} \leq 0, u_{m}^{n}<0
$$


hold for $(m, n) \in Z\left[m_{0}-3 l, m_{0}+2 k-1\right] \times Z\left[n_{0}-3 \delta, n_{0}+1\right]$. By Lemma 3.2 and Lemma 3.1, we have for $m=m_{0}$ and $n=n_{0}$ that

$$
0 \leq \tilde{u}_{m}^{(n+1)}+\underline{a}_{m}^{(n)} \tilde{u}_{m+k}^{(n)}-\bar{b}_{m}^{(n)} \tilde{u}_{m}^{(n-\delta)}-\bar{c}_{m}^{(n)} \tilde{u}_{m-l}^{(n)} \leq\left(-\bar{b}_{m}^{(n)}-\bar{c}_{m}^{(n)}\right) \tilde{u}_{m}^{(n)},
$$

where we have used $\tilde{u}_{m}^{(n+1)}<0, \tilde{u}_{m+k}^{(n)}<0, \underline{a}_{m}^{(n)} \geq 1, \bar{b}_{m}^{(n)} \leq 0$ and $\bar{c}_{m}^{(n)} \leq 0$ for $m=m_{0}$ and $n=n_{0}$. Again, we may see that the inequality (14) leads to a contradiction with (11). The proof is complete.

Theorem 3.4 Suppose there exist constants $\omega_{a}, \omega_{b}, \omega_{c}$ and $\omega$ such that

$$
\mu^{*}(a<1)=\omega_{a}, \mu^{*}(b>0)=\omega_{b}, \mu^{*}(c>0)=\omega_{c}, \mu^{*}(\bar{b} \geq-\bar{c})=\omega,
$$

and

$$
\begin{aligned}
& \mu_{*}((a<1) \cdot(b>0) \cdot(c>0) \cdot(\bar{b} \geq-\bar{c})) \\
& >\frac{\omega_{a}+\omega_{b}+\omega_{c}+\omega}{3}-\frac{1}{3(2 k+3 l)(3 \delta+2)} .
\end{aligned}
$$

Then every nontrivial solution $u=\left\{u_{m}^{(n)}\right\}$ of (2) is frequently oscillatory.

Proof. Suppose to the contrary that $u$ is eventually positive. Then $\mu^{*}(u \leq$ $0)=0$. By Lemma 2.2(iv), we have

$$
\begin{aligned}
& \mu^{*}\left(\Omega-X_{-2 k+1}^{3 l} Y_{-1}^{3 \delta}((a<1)+(b>0)+(c>0)+(\bar{b} \geq-\bar{c})+(u \leq 0))\right) \\
= & 1-\mu_{*}\left(X_{-2 k+1}^{3 l} Y_{-1}^{3 \delta}((a<1)+(b>0)+(c>0)+(\bar{b} \geq-\bar{c})+(u \leq 0))\right) .
\end{aligned}
$$

In view of Lemma 2.3, Lemma 2.2(iii) and (4), we see that the above equality implies that

$$
\begin{aligned}
& \mu^{*}\left(\Omega-X_{-2 k+1}^{3 l} Y_{-1}^{3 \delta}((a<1)+(b>0)+(c>0)+(\bar{b} \geq-\bar{c})+(u \leq 0))\right) \\
\geq & 1-(2 k+3 l)(3 \delta+2)\left\{\mu_{*}((a<1)+(b>0)+(c>0)+(\bar{b} \geq-\bar{c}))+\mu^{*}(u \leq 0)\right\} \\
\geq & 1-(2 k+3 l)(3 \delta+2)\left[\mu^{*}(a<1)+\mu^{*}(b>0)+\mu^{*}(c>0)+\mu^{*}(\bar{b} \geq-\bar{c})\right. \\
& \left.-3 \mu_{*}((a<1) \cdot(b>0) \cdot(c>0) \cdot(\bar{b} \geq-\bar{c}))\right] \\
> & 0 .
\end{aligned}
$$

Thus, by Definition 2.1, we see that

$$
\Omega-X_{-2 k+1}^{3 l} Y_{-1}^{3 \delta}((a<1)+(b>0)+(c>0)+(\bar{b} \geq-\bar{c})+(u \leq 0))
$$

is an infinite subset of $\Omega$. This means that there exists $\left(m_{0}, n_{0}\right) \in \Omega$ with $n_{0}-3 \delta \geq 0$ such that for $(m, n) \in Z\left[m_{0}-3 l, m_{0}+2 k-1\right] \times Z\left[n_{0}-3 \delta, n_{0}+1\right]$,

$$
a_{m}^{(n)} \geq 1, b_{m}^{(n)} \leq 0, c_{m}^{(n)} \leq 0, u_{m}^{(n)}>0
$$


and

$$
\bar{b}_{m}^{(n)}<-\bar{c}_{m}^{(n)}
$$

Similar to the discussions in Theorem 3.3, we may arrive at a contradiction against the very first equality in our proof.

In case $u$ is eventually negative, then $\mu^{*}(u \geq 0)=0$. In an analogous manner, we may see that

$$
\Omega-X_{-2 k+1}^{3 l} Y_{-1}^{3 \delta}((a<1)+(b>0)+(c>0)+(\bar{b} \geq-\bar{c})+(u \geq 0))
$$

is an infinite subset of $\Omega$, which leads to a contradiction again. The proof is complete.

Theorem 3.5 Suppose there exist constants $\omega_{a}, \omega_{b}, \omega_{c}, \omega$ and $\omega_{0} \in(0,1)$ such that

$$
\begin{gathered}
\mu^{*}(a<1)=\omega_{a}, \mu^{*}(b>0)=\omega_{b}, \mu^{*}(c>0)=\omega_{c} \\
\mu_{*}((a<1) \cdot(b>0) \cdot(c>0))=\omega
\end{gathered}
$$

and

$$
\mu_{*}(\bar{b}<-\bar{c})>(2 k+3 l)(3 \delta+2)\left(\omega_{a}+\omega_{b}+\omega_{c}+\omega_{0}-2 \omega\right) .
$$

Then every nontrivial solution $u=\left\{u_{m}^{(n)}\right\}$ of (2) has unsaturated upper positive part.

Proof. In view of Definition 2.5, we only need to prove that $\mu^{*}(u>0) \in$ $(0,1)$. We first assert that $\mu^{*}(u>0)>\omega_{0}$. Otherwise if $\mu^{*}(u>0) \leq \omega_{0}$, then by the same reasoning as in the proof of Theorem 3.3, we have

$$
\left(\Omega-X_{-2 k+1}^{3 l} Y_{-1}^{3 \delta}((a<1)+(b>0)+(c>0)+(u>0))\right) \cdot(\bar{b}<-\bar{c})
$$

is infinite. Then there exists $\left(m_{0}, n_{0}\right) \in \Omega$ with $n_{0}-3 \delta \geq 0$ such that (11) and

$$
a_{m}^{(n)} \geq 1, b_{m}^{(n)} \leq 0, c_{m}^{(n)} \leq 0, u_{m}^{(n)} \leq 0
$$

hold for $(m, n) \in Z\left[m_{0}-3 l, m_{0}+2 k-1\right] \times Z\left[n_{0}-3 \delta, n_{0}+1\right]$. This will lead to a contradiction against $(11)$ as before. Next, we assert that $\mu^{*}(u>0)<1$. Otherwise, $\mu_{*}(u \leq 0)=0$. This is not true in view of the proof of Theorem 3.3. The proof is complete.

Combining Theorem 3.4 and Theorem 3.5, we have the following result and the proof of which is omitted. 
Theorem 3.6 Suppose there exist constants $\omega_{a}, \omega_{b}, \omega_{c}, \omega$ and $\omega_{0} \in(0,1)$ such that

$$
\mu^{*}(a<1)=\omega_{a}, \mu^{*}(b>0)=\omega_{b}, \mu^{*}(c>0)=\omega_{c}, \mu^{*}(\bar{b} \geq-\bar{c})=\omega
$$

and

$$
\begin{gathered}
\mu_{*}((a<1) \cdot(b>0) \cdot(c>0) \cdot(\bar{b} \geq-\bar{c})) \\
>\frac{\omega_{a}+\omega_{b}+\omega_{c}+\omega+\omega_{0}}{3}-\frac{1}{3(2 k+3 l)(3 \delta+2)} .
\end{gathered}
$$

Then every nontrivial solution $u=\left\{u_{m}^{(n)}\right\}$ of (2) has unsaturated upper positive part.

We remark that every nontrivial solution $u$ of (2) has unsaturated lower positive part under the same conditions as Theorem 3.5 or Theorem 3.6.

Next, we consider a two level equation of the form

$$
u_{m}^{(n+1)}+a_{m}^{(n)} u_{m+k}^{(n)}-b_{m}^{(n)} u_{m}^{(n)}-c_{m}^{(n)} u_{m-l}^{(n)}=0,(m, n) \in Z \times N,
$$

where $\left\{a_{m}^{(n)}\right\},\left\{b_{m}^{(n)}\right\},\left\{c_{m}^{(n)}\right\} \in l^{Z \times N}$, and $k, l \in Z[1, \infty)$. Note that (16) is a special case of $(2)$ with $\delta=0$. We have the following results.

Corollary 3.7 Suppose there exists constants $\omega_{a}, \omega_{b}, \omega_{c}$ and $\omega$ such that $\mu^{*}(a<1)=\omega_{a}, \mu^{*}(b>0)=\omega_{b}, \mu^{*}(c>0)=\omega_{c}, \mu_{*}((a<1) \cdot(b>0) \cdot(c>0))=$ $\omega$ and

$$
\mu_{*}(\bar{b}<-\bar{c})>2(2 k+3 l)\left(\omega_{a}+\omega_{b}+\omega_{c}-2 \omega\right) .
$$

Then every nontrivial solution $u=\left\{u_{m}^{(n)}\right\}$ of (16) is frequently oscillatory.

Corollary 3.8 Suppose there exists constants $\omega_{a}, \omega_{b}, \omega_{c}$ and $\omega$ such that $\mu^{*}(a<1)=\omega_{a}, \mu^{*}(b>0)=\omega_{b}, \mu^{*}(c>0)=\omega_{c}, \mu^{*}(\bar{b} \geq-\bar{c})=\omega$ and

$$
\mu_{*}((a<1) \cdot(b>0) \cdot(c>0) \cdot(\bar{b} \geq-\bar{c}))>\frac{\omega_{a}+\omega_{b}+\omega_{c}+\omega}{3}-\frac{1}{6(2 k+3 l)} .
$$

Then every nontrivial solution $u=\left\{u_{m}^{(n)}\right\}$ of (16) is frequently oscillatory. 
Corollary 3.9 Suppose there exists constant $\omega_{a}, \omega_{b}, \omega_{c}, \omega$ and $\omega_{0} \in(0,1)$ such that

$$
\begin{gathered}
\mu^{*}(a<1)=\omega_{a}, \mu^{*}(b>0)=\omega_{b}, \mu^{*}(c>0)=\omega_{c} \\
\mu_{*}((a<1) \cdot(b>0) \cdot(c>0))=\omega
\end{gathered}
$$

and

$$
\mu_{*}(\bar{b}<-\bar{c})>2(2 k+3 l)\left(\omega_{a}+\omega_{b}+\omega_{c}+\omega_{0}-2 \omega\right) .
$$

Then every nontrivial solution $u=\left\{u_{m}^{(n)}\right\}$ of (16) has unsaturated upper positive part.

Corollary 3.10 Suppose there exists constants $\omega_{a}, \omega_{b}, \omega_{c}, \omega$ and $\omega_{0} \in(0,1)$ such that

$$
\mu^{*}(a<1)=\omega_{a}, \mu^{*}(b>0)=\omega_{b}, \mu^{*}(c>0)=\omega_{c}, \mu^{*}(\bar{b} \geq-\bar{c})=\omega,
$$

and

$\mu_{*}((a<1) \cdot(b>0) \cdot(c>0) \cdot(\bar{b} \geq-\bar{c}))>\frac{\omega_{a}+\omega_{b}+\omega_{c}+\omega+\omega_{0}}{3}-\frac{1}{6(2 k+3 l)}$.

Then every nontrivial solution $u=\left\{u_{m}^{(n)}\right\}$ of (16) has unsaturated upper positive part.

\section{An Example}

We give an example to illustrate our previous results. Consider the discrete heat equation (2) with $a_{m}^{(n)}=1$ and $b_{m}^{(n)}=c_{m}^{(n)}=-1$ on $(m, n) \in Z \times N$, that is,

$$
u_{m}^{(n+1)}+u_{m+1}^{(n)}-u_{m}^{(n-1)}-u_{m-1}^{(n)}=0,(m, n) \in Z \times N .
$$

It is clear that

$$
\begin{gathered}
\mu_{*}(\bar{b}<-\bar{c})=1 \\
\mu^{*}(a<1)=\mu^{*}(b>0)=\mu^{*}(c>0)=\mu^{*}(\bar{b} \geq-\bar{c})=0
\end{gathered}
$$

as well as

$$
\mu_{*}((a<1) \cdot(b>0) \cdot(c>0))=\mu_{*}((a<1) \cdot(b>0) \cdot(c>0) \cdot(\bar{b} \geq-\bar{c}))=0 .
$$

Hence by Theorem 3.3 or Theorem 3.4, every nontrivial solution $u$ of (17) is frequently oscillatory. Furthermore, let $\omega_{0} \in(0,1 / 25)$, we see that all conditions in Theroem 3.5 or Theorem 3.6 are satisfied. Consequently, every nontrivial solution $u$ of (17) has unsaturated upper positive part. As a matter of fact, $u=\left\{(-1)^{m} 2^{n-m}\right\}_{(m, n) \in Z \times N}$ is such a solution of $(17)$ with $\mu^{*}(u>0)=1 / 2$. 


\section{References}

[1] S. S. Cheng and G. H. Lin, Green's function and stability of a linear partial difference scheme, Computers Math. Applic., 35(1998), 27-41.

[2] S. S. Cheng, Partial Difference Equations, Taylor and Francis, 2003.

[3] I. Niven, The asymptotic density of sequences. Bull. Amer. Math. Soc. 57, (1951). 420-434.

[4] A. R. Freedman, A. R. On asymptotic density in $n$-dimensions, Pacific J. Math. 29(1969), 95-113.

[5] C. J. Tian, S. L. Xie and S. S. Cheng, Measures for oscillatory sequences, Comput. Math. Applic. 36 (1998), 149-161.

[6] C. J. Tian and B. Q. Zhang, Frequent oscillation of a class of partial difference equations, Journal for Analysis and its Applications, 18(1)(1999), 111-130.

Received: November 29, 2005 